\title{
A Simple and Portable Reader to Analyze Concentration of Iron in Blood Plasma via Colorimetric Analysis
}

\author{
Siti Nur'Arifah Binti M.Soair ${ }^{1 *}$, Arthur Foo Yen $\operatorname{Tan}^{2}$, Boon Kar Yap ${ }^{3}$ \\ ${ }^{1,2,3}$ Electronics and Communication Department, College of Engineering, Universiti Tenaga Nasional, KM-7 Jalan Uniten-Ikram, 43000 \\ Kajang, Selangor Malaysia \\ *Corresponding author E-mail: sitinurarifah.msoair@gmail.com
}

\begin{abstract}
This paper presents a simple and portable body iron reader to analyze the concentration of iron via colorimetric analysis. Through this approach, early diagnosis can be made for diseases such as iron deficiency anemia (IDA) and thalassemia. The uses of spectrophotometer to analyze a concentration of analyte in a sample can be reconsidered. Spectrophotometer nowadays is bulky and very expensive. The proposed portable reader can gives the user a result of concentration of analyte in a sample solution almost the same as the commercially available spectrophotometer. Through a comparison, the portable body iron reader managed to obtain a 0.9986 coefficient of determination value which has only $0.13 \%$ of error. Moreover, this device can works with a battery without the needs of power source.
\end{abstract}

Keywords: Iron Deficiency Anemia; Light Absorbance; Photometer; Spectrophotometer

\section{Introduction}

Blood plasma stores a lot of proteins that resemble the health condition of a person. One of the most important compositions in blood plasma is iron [1]. As an important part of hemoglobin, a body needs the iron for it to function normally. Hemoglobin needs iron to make new red blood cells. And the remaining will be stored as a support in the body. Iron deficiency anemia (IDA) and thalassemia are the two examples of disease that required them to know the status of body iron concentration in an early state. The gold standard procedure to access body iron concentration is through the bone marrow iron [2]. However, this is a very risky procedure thus a different approach is used which is measuring the plasma iron (iron that is responsible to transfer proteins in plasma) [3]. Moreover, the sophisticated machine and instruments to diagnose IDA are bulky and expensive.

Colorimetric analysis has been widely used in a device to determine the concentration of a chemical substance using color reagents. The basis of colorimetric analysis is when a specific color of light passes through a solution, the solution absorbs a part of the light [4]. To obtain a maximum light absorbance, there is a specific color of the solution that varies according to the range of the wavelength. An instrument that commonly uses colorimetric analysis is called colorimeter and it works on a specific wavelength [5].

To date, various works have been reported on developing low-cost colorimeters [6-9]. Most of the researchers nowadays use a microcontroller to simplify the process of controlling and measuring the colorimetric devices [5, 6, 10, 11]. A simple and portable single channel colorimeter was developed by Suzuki et al. to determine the nitrite and iron in river water [10]. Kittipannyangam et al. reported a low-cost battery-powered light absorbance device to be used in analytical measurements [4]. Their device utilized the uses of red, green and blue light emitting diode (LED) despite the usage of monochromator or wavelength selective filter as it can reduce space. Another device reported for analytical measurement is the simple, low power; inexpensive and microcontroller-based (SLIM) spectrometer by Cantrell et al. that uses thionine as an analyte [12]. A few dual channel colorimeter has also been reported to be used in $\mathrm{pH}$ seawater measurement, chemical instrumentation education and measuring chemical oxygen demand (COD) to reduce errors and improve the device sensitivity $[7,8,11-13]$. Despite all the colorimeter devices that have been developed, no specific colorimeter is being developed for measuring the concentration of iron in blood plasma. Thus, a portable colorimeter or body iron detection circuit using colorimetric analysis is presented in this study. The colorimeter is not only portable, it is compact $(134.7 \times 89.9) \mathrm{mm}$, and can be powered on using a battery.

In this paper, we describe the system design including the sample preparation and circuit design of the iron detection circuit. Colorimetric analysis and the result are presented. The device has a potential to be a low-cost colorimeter for detection of iron concentration in blood plasma, especially in poor and rural areas.

\section{Materials and Methods}

\subsection{System and Circuit Design}

Fig. 1 shows the block diagram of the body iron store reader. When the LED passes through a cuvette containing the plasma that has been mixed with reagent, the photodetector detects the transmitting light. Photodetector then passes the signal to the microcontroller for light absorbance calculation. The output of the microcontroller is then displayed on the liquid-crystal display (LCD). 


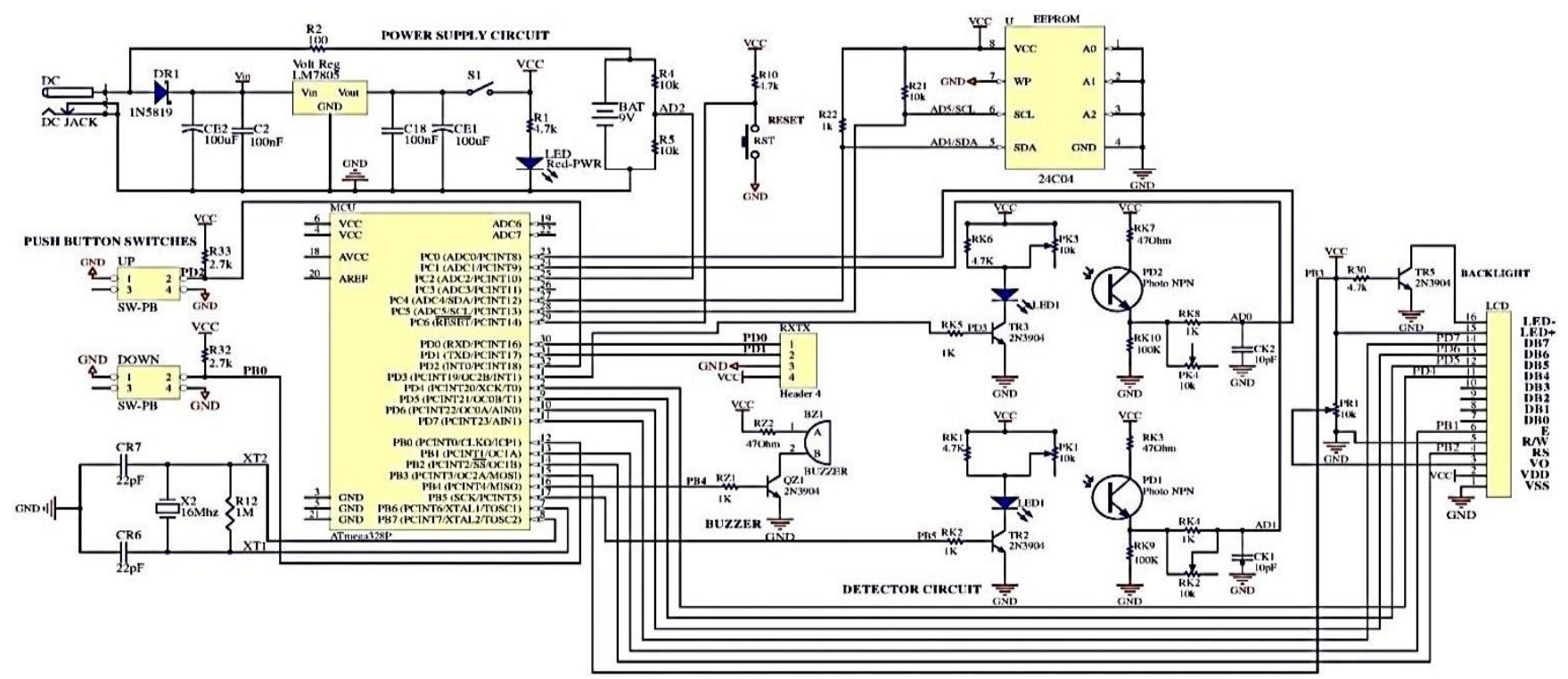

Fig. 1: Complete circuit configuration

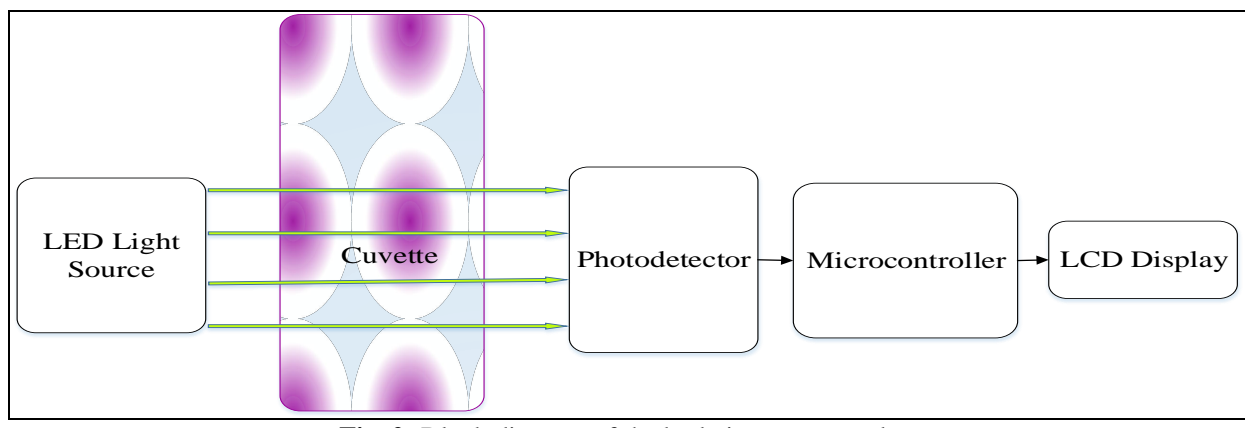

Fig. 2: Block diagram of the body iron store reader.

Fig. 2 illustrates the complete circuit diagram of the system. LED model of Marubeni L560-06 is chosen as the light source. With spectral emission of $560 \mathrm{~nm}$, it has the colour of yellow-green. The cuvette used in the experiment is a standard size cuvette of $(45 \mathrm{x}$ $10 \times 10) \mathrm{mm}$ that requires a sample volume of $1 \mathrm{~mL}$. Light is detected by TEPT5700 NPN phototransistor purchased from Vishay Semiconductors. It consists of an NPN phototransistor, potentiometer, capacitor and resistor. With a spectral range of $440 \mathrm{~nm}$ to $800 \mathrm{~nm}$, it has a peak of $570 \mathrm{~nm}$ which is very close to the LED spectral emission. The phototransistor has two terminals which are collector and emitter. The output is obtained from emitter. The operations are controlled by ATmega328P MCU. All the inputs and outputs are controlled here and it is an 8-bit RISC-based MCU. The output is then passed through a 10-bit analogue to digital converter (ADC) before it is being processed displayed on the LCD screen. The software used to simulate this program is via Arduino UNO model and circuit design was modelled in Proteus Design Suite.

\subsection{Samples Preparation}

Real human plasma is not used in this experiment. Instead, a laboratory-prepared iron solution that contains similar types of ion as in plasma is used. The solution is called revised simulated body fluids (r-SBF). R-SBF is then used as a solution that mimicking human plasma environment and mixed with reagent in order to determine the iron level. Five different reagents were used in order to prepare the samples; citric acid (CA), thiourea (TU), ascorbic acid (AsC), ferrozine (FRZ) and ferrous $\left(\mathrm{Fe}^{2}\right)$. Figure 2 shows the 12 cuvettes containing samples with different iron concentration. Two of the samples are blank samples which are samples without iron as the reference. The rest of the samples contain iron concentration ranging from 50 to $4000 \mu \mathrm{g} / \mathrm{L}$.

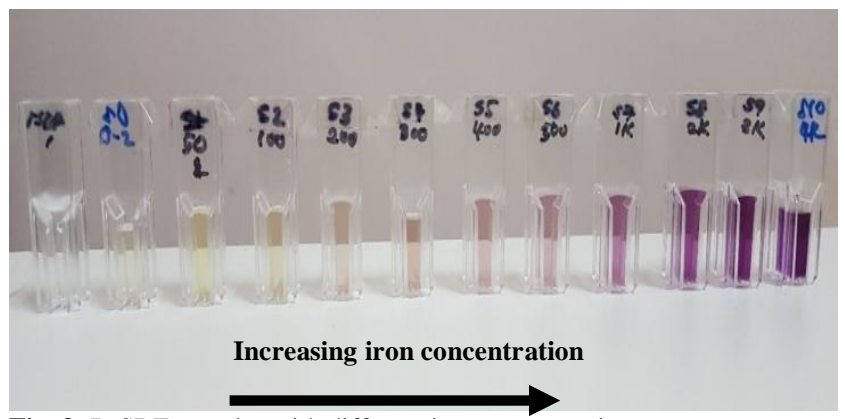

Fig. 3: R-SBF samples with different iron concentration

\subsection{Light Absorbance}

Light absorbance of the sample can be determined by the Beer Lambert's Law as in (1).

$A=-\log \frac{I}{n}=\varepsilon c l$

Where $A$, the absorbance have the constant of proportionality to the molar absorptivity, $\varepsilon$, concentration of the compound, $c$ and the path length of the sample, $l . I$ and $I_{\circ}$ refer to the transmitted light and incident light intensity respectively [15]. However, absorbance of the light cannot be measured directly. Instead, output of the photometer comes out in a form of voltage. When light passes through the cuvette, other processes also tend to decrease the intensity of the light. In that case, taking the reference reading 
that correspond to the light intensity is necessary. This can be done by passing the light through a sample without reagent known which is the blank sample. The circuit does not give $0 \mathrm{~V}$ output reading when no light pass through. Due to this error, correction needs to be made by eliminating voltage reading at zero light

$\left(V_{\text {zero }}\right)$ from all the sample readings as in (2).

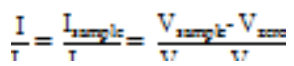

Based on equation (1) and (2), the absorbance equation can be simplified as in (3).

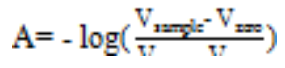

\subsection{Coefficient of Determination}

Coefficient of determination, $\mathrm{R}^{2}$ is a number that can show how good a series of data used in an experiment fits to a statistical model [15]. Ranging from 0 to 1 , the most good relationship is when the value of $\mathrm{R}^{2}$ nearest to 1 . In statistics, this is how they measure the successfulness level of an outcome to a model. In this experiment, the coefficient of determination is used to measure the relationship value between the light absorbance and the solution concentration. $R^{2}$ can be measured through the equation in (4).

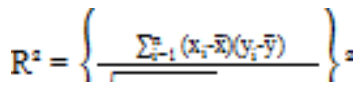

Where $\boldsymbol{n}$ refer to the number of solution, $\boldsymbol{x}_{\tilde{i}}$ and $\boldsymbol{y}_{\tilde{i}}$ refer to the light absorbance and the concentration of solution respectively. Lastly, $\overline{\mathbf{x}}$ and $\overline{\mathrm{y}}$ refer to the mean of light absorbance and mean of the concentration of the solution.

\subsection{Linear Regression}

From Beer Lambert's law [4], linear regression method is always used to determine unknown concentration of a solution. This is by using the light absorbance of the solution. It is used to model the relationship between $y$, the concentration of the solution to $X$, the light absorbance (5). While $a$ is a constant value when $X$ is 0 and $b$ is slope.

$$
y=a+b x
$$

\section{Experimental Result}

\subsection{Maximum Absorbance Wavelength}

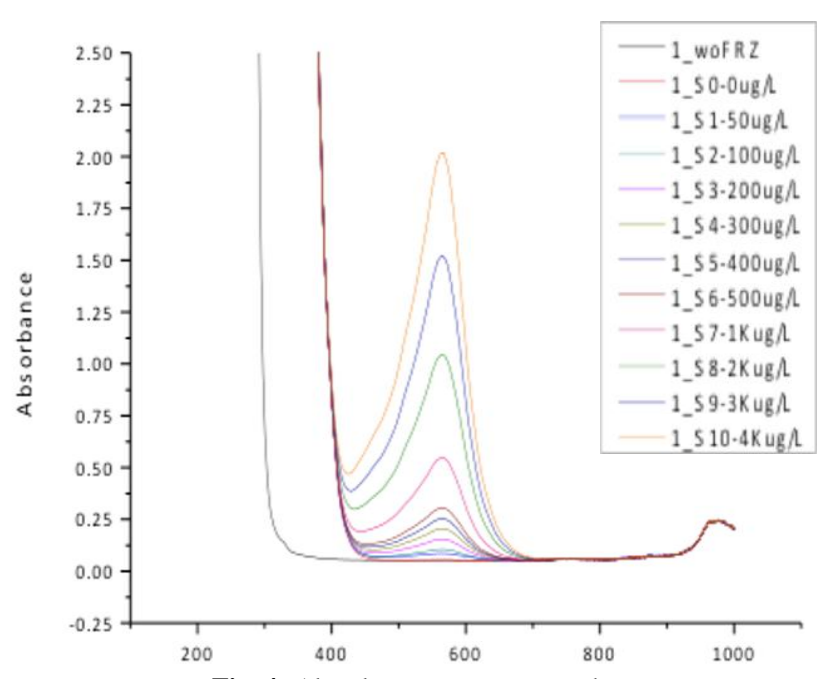

Fig. 4: Absorbance spectrum graph

Fig. 4 shows the absorbance spectrum graph of the samples that have different iron concentrations ranging from $0 \mu \mathrm{g} / \mathrm{L}$ to 4000 $\mu \mathrm{g} / \mathrm{L}$. the blank sample is labelled as woFRZ. Y-axis of the graph refers to absorbance and the $\mathrm{x}$-axis refers to the wavelength. The graph have the bell-shaped curve denotes that the absorbance increases when the wavelength increases (400nm to $562 \mathrm{~nm}$ ). Accordingly, as the wavelength goes from $562 \mathrm{~nm}$ to $700 \mathrm{~nm}$, the absorbance decreases. The graph proves that the peak of the wavelength is at $562 \mathrm{~nm}$. It also concludes that as the concentration of the iron increases, the light absorbance of the solution increases.

\subsection{Linear Regression Graph}

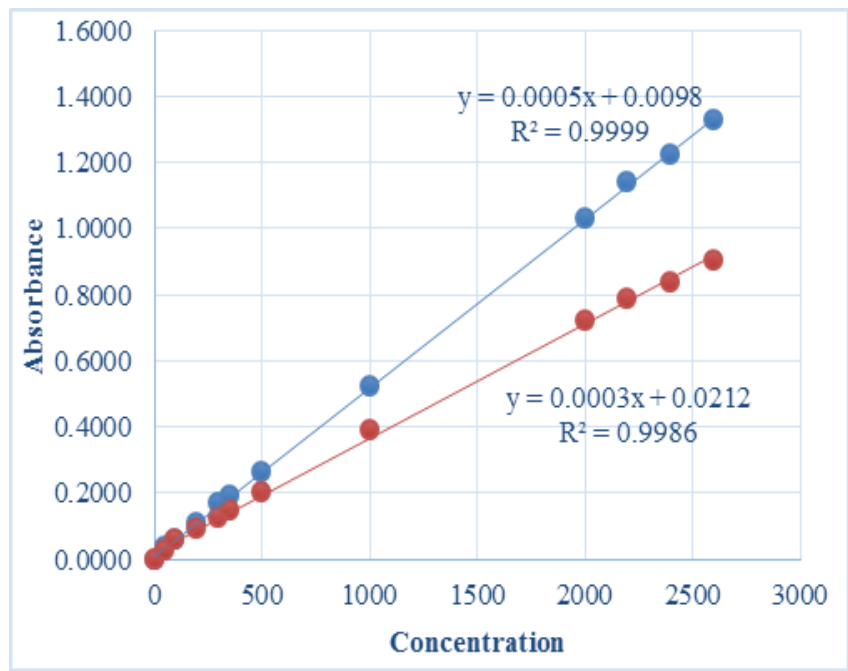

Fig. 5: Calibration curve comparison between spectrophotometer and the body iron reader.

In our experiment, the result obtained is compared with the commercially available spectrophotometer machine to know the efficiency. Fig. 5 shows the plots of calibration curve for the spectrophotometer and the body iron reader. Both of the instruments fulfilled the Beer Lambert's law as they can produce a straight line. The straight line is plotted between the concentrations of the solution to the absorbance and calculated using the linear regression relationship. $\mathrm{R}^{2}$ is the coefficient of the determination. The straight line in blue indicates the calibration curve for the spectrophotometer that have the gradient of 0.9999 . While the body iron reader have the gradient of 0.9986 which is shown in the red line. The values show that the gradient of the body iron reader is very close to the gradient of commercially available spectrophotometer. As the maximum value for $\mathrm{R}^{2}$ is 1 , the percentage error between them is only $0.13 \%$. This proves that the reader is suitable to be used for analytical chemistry. 


\subsection{Iron Stages Status}

The body portable iron reader is used to determine the level of iron status in a body of a patient. Samples were prepared to have a reaction towards the ferrous, Fe. Different amount of serum ferritin (serum $\mathrm{Fe}$ ) are used in order to determine the status of iron as in Table 1. Five different statuses are used namely, deficiency, negative balance, normal, positive balance, and overload. The corresponding iron concentrations are $350 \mu \mathrm{g} / \mathrm{L}, 461 \mu \mathrm{g} / \mathrm{L}, 1039$ $\mu \mathrm{g} / \mathrm{L}, 1879 \mu \mathrm{g} / \mathrm{L}$, and $2600 \mu \mathrm{g} / \mathrm{L}$ respectively.

Table 1: Serum concentrations level for different iron stages. Adopted and modified from [16].

\begin{tabular}{|c|c|}
\hline Stage of Iron Status & Serum Fe $(\mu \mathrm{g} / \mathrm{L})$ \\
\hline Deficiency & 350 \\
\hline Negative Balance & 461 \\
\hline Normal & 1039 \\
\hline Positive Balance & 1879 \\
\hline Overload & 2600 \\
\hline
\end{tabular}

\section{Conclusion}

A simple and portable spectrophotometer to be used as body iron reader has been proposed in this paper. The portable iron reader could help in solving problems in detecting patients with anaemic diseases in an early stage, especially for the people living in poor countries and rural areas. This device works in a simple way and most of the general parts are affordable. By using the colorimetric analysis method, the concentration of iron in a sample solution can be determined. The yellowish-green LED which has a spectral emission of $560 \mathrm{~nm}$ has an error of less than $0.5 \%$ compared to the real spectrophotometer. In addition, the proposed device has a coefficient of determination, $\mathrm{R}^{2}$ value of 0.0086 which is very close to the $\mathrm{R}^{2}$ value of the spectrophotometer. This proves that the device is capable of working for analytical measurement.

\section{Acknowledgement}

This work is fully funded by Malaysia ministry of higher education (MOHE) - 20170101PRGS.

\section{References}

[1] W.N.M. Ramsay, PLASMA IRON, vol. 1. 1958.

[2] R. DOROTHY SUNDBERG and HARRIETTE BROMAN, "The Application of the Prussian Blue Stain to Previously Stained Films of Blood and Bone Marrow," Blood, vol. 10, no. 2, pp. 160-166, 1955 .

[3] B. J. Bain, S. M. (Shirley M. Lewis, and J. V. (John V. Dacie, Dacie and Lewis practical haematology. Elsevier Churchill Livingstone, 2012

[4] S. Kittipanyangam, K. Abe, and K. Eguchi, "Design of a measurement device explaining the relationship between the concentration of solution and the light absorbance for chemical education," 2016 13th Int. Conf. Electr. Eng. Comput. Telecommun. Inf. Technol. ECTI-CON 2016, 2016.

[5] A. F. Y. Tan, N. A. Talik, B. K. Yap, and P. S. Krishnan, "Prospects of Low-Cost Photometers for Colorimetric Serum Iron Concentration Determination," Springer, Singapore, 2018, pp. 165169.

[6] A. Udoji Itodo, A. Usman, S. Bashir Sulaiman, and H. Ugbede Itodo, "Color Matching Estimation ofIron Concentrationsin Branded Iron Supplements Marketed in Nigeria," Adv. Anal. Chem. Sci. Acad. Publ., vol. 2, no. 1, pp. 16-23, Aug. 2012.

[7] G. C. Anzalone, A. G. Glover, and J. M. Pearce, "Open-source colorimeter," Sensors (Switzerland), vol. 13, no. 4, pp. 5338-5346, 2013.

[8] N. B. Jones, C. Riley, M. S. Sheya, and M. M. Hosseinmardi, "A low-cost colorimeter," J. Med. Eng. Technol., vol. 8, no. 1, pp. 9 14, Jan. 1984.
[9] J.-S. Kim et al., "Simple LED spectrophotometer for analysis of color information," Biomed. Mater. Eng., vol. 26, no. s1, pp. S1773-S1780, Aug. 2015.

[10] M. Suzuki, Y., Aruga, T., Kuwahara, H., Kitamura, M., Kuwabara T., Kawakubo, S., Iwatsuki, "A simple and portable colorimeter using a red-green-blue light-emitting diode and its application to the on-site determination of nitrite and iron in river-water,' Analytical-sciences-the-international-journal-of-the-Japan-Societyfor-Analytical-Chemistry, vol. 20, no. 6, pp. 975-977, 2004.

[11] B. Yang, M. C. Patsavas, R. H. Byrne, and J. Ma, "Seawater pH measurements in the field: A DIY photometer with 0.01 unit $\mathrm{pH}$ accuracy," Mar. Chem., vol. 160, pp. 75-81, 2014.

[12] K. M. Cantrell and J. D. Ingle, "The SLIM spectrometer," Anal. Chem., vol. 75, no. 1, pp. 27-35, 2003.

[13] P. K. Bhadane, "Development of Microcontroller Based Analyzer for the Detection of Chlorine in Water," Int. J. Innov. Res. Sci. Eng. Technol., vol. 3, no. 4, pp. 10890-10897, 2014.

[14] J. P. Grinias, J. T. Whitfield, E. D. Guetschow, and R. T. Kennedy, "An inexpensive, open-source USB Arduino data acquisition device for chemical instrumentation," J. Chem. Educ., vol. 93, no. 7, pp. 1316-1319, 2016.

[15] STEWART J. TAVENER, "Build your own spectrophotometer Feature | Education in Chemistry," Educ. Chem., pp. 151-154, 2007.

[16] V. Herbert et al., "Serum Ferritin Iron, a New Test, Measures Human Body Iron Stores Unconfounded by Inflammation," Stem Cells, vol. 15, no. 4, pp. 291-296, Jul. 1997. 